\title{
MEDITERRANEAN DIET AND ADOLESCENTS WITH INTELLECTUAL DISABILITIES
}

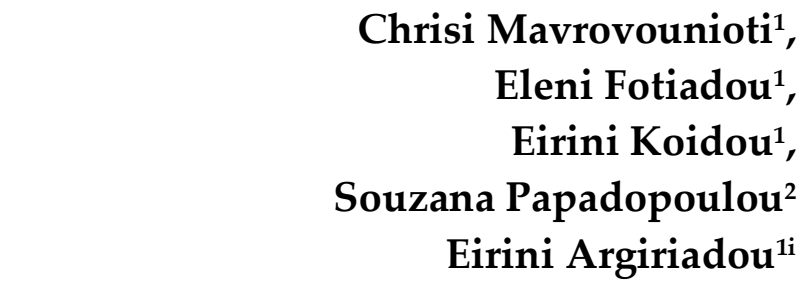

1School of Physical Education and Sport Sciences, Aristotle University of Thessaloniki, Greece 2Department of Nutritional Sciences and Dietetics, School of Health Sciences, International Hellenic University, Greece

\begin{abstract}
:
The purpose of the present study is to evaluate the adoption of Mediterranean diet in adolescents with intellectual disabilities. The sample of the study consisted of 24 adolescents ( 8 boys and 16 girls) with intellectual disabilities who were studying at a secondary education school, with an average age of $17.63+2.018$ years. For data collection, the Mediterranean Diet Quality Index for Children and Adolescents (KIDMED) by SerraMajem et al. (2004) was used. KIDMED is supported by specific principles sustaining traditional healthy Mediterranean dietary patterns. KIDMED is based on a 16-item food consumption log questionnaire that can be answered either self-administered or in interview. As for the pupils with intellectual disabilities, the answers were recorded in an individual interview. For the statistical analysis the Statistical Package for Social Sciences ver. 23.0 for windows was used. Data processing showed that over the 1/3 of adolescents with intellectual disabilities (37.5\%) has a very low diet quality, while the proportion of adolescents following an optimal Mediterranean diet was zero. In addition, the diet of the adolescents with intellectual disabilities in a very large proportion (62.5\%) needs improvement for the adaptation of the consumption / intake to Mediterranean patterns. The results show that adolescents with intellectual disabilities do not follow the Mediterranean diet. Therefore, priority should be given to the application and implementation of health educational programs on diet in general, and more specifically on the benefits of Mediterranean diet to adolescents with intellectual disabilities, in order
\end{abstract}

i Correspondence: email argiriadoue@phed-sr.auth.gr 
to adopt the Mediterranean diet patterns aiming on the beneficial effects, both directly and long-term in their adult life.

Keywords: youth, mental retardation, food, diet quality index

\section{Introduction}

The term "Mediterranean diet" reflects the characteristics of dietary patterns of several countries in the Mediterranean Basin during the early 1960s (Serra-Majem et al., 2004). The Mediterranean diet is a good for the health and well-balanced diet, that provides most of the nutrients in their right proportions (Ríos-Hernández et al., 2017).

More specifically, the Mediterranean dietary pattern has its base in high consumption of vegetables and fruits, cereals and whole grains, pulses as source of dietary fibre, nuts, fish, olive oil as the main added fat, and low-fat dairy products. Additionally, it is noted that there is a relationship between the Mediterranean dietary pattern and reduced risk of the most common chronic degenerative diseases. On the contrary, increased total calorie intake, overconsumption of animal fat and (red) meat, sugar and processed food, as well as body weight, and especially excess body fat, were found to be associated with a greater risk of the most common chronic degenerative diseases (Donini et al., 2015). It is worth to be mentioning that obesity alone presents a higher risk of developing many chronic conditions, such as hypertension, diabetes, heart disease, arthritis, stroke, stress, depression, respiratory diseases and even sleep disorders (Ells et al, 2006; Haveman et al, 2010).

Concerning people with intellectual disability (ID), they confront a significant health problem as they are more overweight or obese in comparison with people of the general population in a rate higher by 59\% (Marshall et al, 2003; Draheim, 2006). The causes are multifactorial and dependent on a number of factors, such as hypotonia, endocrine abnormalities depressed metabolic rate, reduced exercise, and mainly poor eating behaviours and high inappropriate caloric eating (Mokdad et al, 2004; Fisher and Ketti, 2005). Concerning young people with ID, they have a higher risk of developing health disorders related to obesity, earlier in their life, because they present high levels of obesity (Janiciki et al, 2002).

Unfortunately, healthy eating aiming on health promotion does not adopted early in the life, but begins only when obesity is already a clearly perceptible problem for the people with intellectual disability. However, at this point of time, every day diet habits that resulted to obesity are well established and the process to re-educate the subjects is more difficult (Jobling, 2001).

So, given the vulnerability of young people with ID regarding obesity mainly due to poor diet, it is understood, that more studies are needed, which may provide more data overeating behaviors of young people with ID. However, despite the observed sufficient number of studies on eating behaviors of people of general population there is a lack of studies examining the adherence to the Mediterranean Diet and almost no study examining the adherence to the Mediterranean Diet of the adolescents with ID in Greece. 
Thus, the aim of the present study is to examine the adherence to the MED of adolescents with ID.

\section{Methods}

\subsection{Sample}

Twenty-four adolescents with ID (eight boys and sixteen girls) volunteered to participate in the research. All the subjects were pupils attending courses in a secondary education school for pupils with ID in Greece. During the conduction of the survey, the school year was in progress. Subjects' age ranged from 13 to 21 years $(\mathrm{M}=17.63, \mathrm{SD}=2.018)$.

\subsection{Procedure}

An approval for conducting the research was given by the director of the school, after the aim and the conditions of the research were described. The procedures were in agreement with the ethical standards of the Declaration of Helsinki of the World Medical Association (2000).

Before the beginning of the research, a written informed consent for the participation in the research was obtained from the parents or guardians of each pupil. In continuity, a description of general requirements, in a simple and understandable way for the pupils was given. The need for absolute honesty and precision was particularly emphasized. Subsequently all the pupils answered to a questionnaire once, in an individual interview with the researcher, during which simple instructions were given and pupils' questions were explained. Additionally, measurements of anthropomorphological characteristics, such as height and weight were carried out.

\subsection{Scale of Measurements}

For data collection, the Mediterranean Diet Quality Index for children and adolescents (KIDMED) of Serra-Majem and his colleagues (2004) was used. The KIDMED index is used for the evaluation of adherence in Mediterranean diet. The KIDMED is based on certain principles sustaining and challenging traditional healthy Mediterranean dietary patterns.

The KIDMED index was inspired by instruments previously developed for adult (Alberti-Fidanza, \& Fidanza, 2004) and elderly (Trichopoulou et al., 1995) populations. Thus, the KIDMED index, consist the first index evaluating the adequacy of Mediterranean dietary patterns in children and youth that is for population aged 2-24 years.

The KIDMED was based on a 16-question test that could be self-administered or conducted by interview (teacher, pediatrician, dietitian, etc.). As for the pupils with ID, the answers were recorded personally for each subject by interview with the researcher, who is pupils' teacher. KIDMED index ranged from -4 to 12 degrees. Questions denoting a negative connotation with respect to the Mediterranean diet were assigned a value of 1. Questions denoting a positive aspect were assigned a value of +1 (see Table 1 ). 
Table 1: KIDMED test to assess the Mediterranean diet quality

\begin{tabular}{|c|l|}
\hline \multicolumn{2}{|l|}{ Scoring } \\
\hline+1 & I take a fruit or a fruit juice every day \\
\hline+1 & I have a second fruit every day \\
\hline+1 & I have fresh or cooked vegetables regularly once a day \\
\hline+1 & I have fresh or cooked vegetables more than once a day \\
\hline+1 & I consume fish regularly (at least 2-3 times per week) \\
\hline-1 & I go more than once a week to a fast-food (hamburger) restaurant \\
\hline+1 & I like pulses and eats them more than once a week \\
\hline+1 & I consume pasta or rice almost every day (5 or more times per week) \\
\hline+1 & I have cereals or grains (bread, etc.) for breakfast \\
\hline+1 & I consume nuts regularly (at least 2-3 times per week) \\
\hline+1 & We use olive oil at home \\
\hline-1 & I skip breakfast \\
\hline+1 & I have a dairy product for breakfast (yoghurt, milk, etc.) \\
\hline-1 & I have commercially baked goods or pastries for breakfast \\
\hline+1 & I take two yoghurts and/or some cheese (40 g) daily \\
\hline-1 & I take sweets and candy several times every day \\
\hline
\end{tabular}

The sums of the values from the administered test were classified into three categories/levels:

1. $\geq 8$ : optimal Mediterranean diet;

2. 4-7: improvement needed to adjust intake to Mediterranean patterns;

3. $\leq 3$ : very low diet quality.

Thus, higher scores in KIDMED show a higher adherence in Mediterranean diet.

\subsection{Statistical Analyses}

For data analysis the SPSS ver. 23.0 for Windows was used. Descriptive analysis and frequencies were applied. Descriptive statistics were used for participants' profile analysis and variable description. Results were expressed as mean values, standard deviation, and percentages.

\section{Results}

In Table 2, the anthropomorphological characteristics of the sample are presented.

Table 2: Sample's anthropomorphological characteristics

\begin{tabular}{|l|c|c|}
\hline & Mean & Standard Deviation \\
\hline Age $($ years $)$ & 17.63 & 2.018 \\
\hline Height $(\mathrm{cm})$ & 165.29 & 8.18 \\
\hline Body Weight $(\mathrm{Kg})$ & 71.13 & 17.23 \\
\hline BMI $\left(\mathrm{Kg} / \mathrm{m}^{2}\right)$ & 26.09 & 6.23 \\
\hline
\end{tabular}

In Table 3, pupils' frequencies of adherence of the principles sustaining traditional healthy Mediterranean dietary patterns are presented. 
Table 3: Frequencies of adherence of the principles sustaining traditional healthy Mediterranean dietary patterns

\begin{tabular}{|l|c|c|}
\hline & Adherence \\
\hline Principles sustaining traditional healthy Mediterranean dietary patterns & $\begin{array}{c}\text { YES } \\
\%\end{array}$ & $\begin{array}{c}\text { NO } \\
\%\end{array}$ \\
\hline Fruit or fruit juice daily & $87.5 \%$ & $12.5 \%$ \\
\hline Second serving of fruit daily & $75 \%$ & $25 \%$ \\
\hline Fresh or cooked vegetables daily & $79.2 \%$ & $20.8 \%$ \\
\hline Fresh or cooked vegetables $>1$ time/day & $62.5 \%$ & 37.5 \\
\hline Regular fish consumption (at least 2-3 times/week) & $0 \%$ & $100 \%$ \\
\hline$>1$ time/week fast-food (hamburger) restaurant & $62.5 \%$ & $37.5 \%$ \\
\hline Pulses $>1$ time/week & $91.7 \%$ & $8.3 \%$ \\
\hline Pasta or rice almost daily (>5 times/week) & $83.3 \%$ & $16.7 \%$ \\
\hline Cereal or cereal product for breakfast & $12.5 \%$ & $87.5 \%$ \\
\hline Regular nut consumption (at least 2-3 times/week) & $0 \%$ & $100 \%$ \\
\hline Use of olive oil at home & $95.8 \%$ & $4.2 \%$ \\
\hline Skip breakfast & $25 \%$ & $75 \%$ \\
\hline Dairy product for breakfast & $37.5 \%$ & $62.5 \%$ \\
\hline Commercially baked goods or pastries for breakfast & $62.5 \%$ & $37.5 \%$ \\
\hline Two yoghurts and/or 40 gr cheese daily & $12.5 \%$ & $87.5 \%$ \\
\hline Sweets and candy several times a day & $70.8 \%$ & $29.2 \%$ \\
\hline
\end{tabular}

As it is shown in Table 3, and concerning eating habits denoting a positive connotation with respect to the Mediterranean diet, the pupils act positively in seven of them and negatively in the other five. More specifically, pupils' bigger percentages take a fruit or a fruit juice every day $(87.5 \%)$, have a second fruit every day $(75 \%)$, have fresh or cooked vegetables regularly once a day $(79.2 \%)$ or/and more than once a day $(62.5 \%)$, eat pulses more than once a week $(91.7 \%)$, consume pasta or rice almost every day (5 or more times per week) (83.3\%) and use olive oil at home (95.8\%). However, pupils with ID in the present study do not consume fish and nuts regularly that is at least 2-3 times/week, and their bigger percentage $(87.5 \%)$ do not consume cereals or cereal products for breakfast and two yoghurts and/or 40 gr cheese daily. Moreover, pupils' bigger percentage (62.5\%) do not consume dairy products for breakfast.

As for eating habits denoting a negative connotation with respect to the Mediterranean diet, unfortunately, the pupils act positively in the most of them. More specifically, the bigger percentage consume sweets and candy several times a day $(70.8 \%)$, consume commercially baked goods or pastries for breakfast $(62.5 \%)$, and eat more than 1 time/week at a fast-food (hamburger) restaurant (62.5\%). Fortunately, only $25 \%$ of the pupils skip the breakfast.

Thus, the pupils follow only half of the principles sustaining traditional healthy Mediterranean dietary patterns in percentages from $62.5 \%$ to $95.8 \%$. Consequently, it could be said that pupils' percentages of adherence of the principles sustaining traditional healthy Mediterranean dietary patterns are very low or/and zero.

In Figure 1 are presented the frequencies of the pupils as they were classified into the three categories/levels according to the sums of the values from the KIDMED test. 


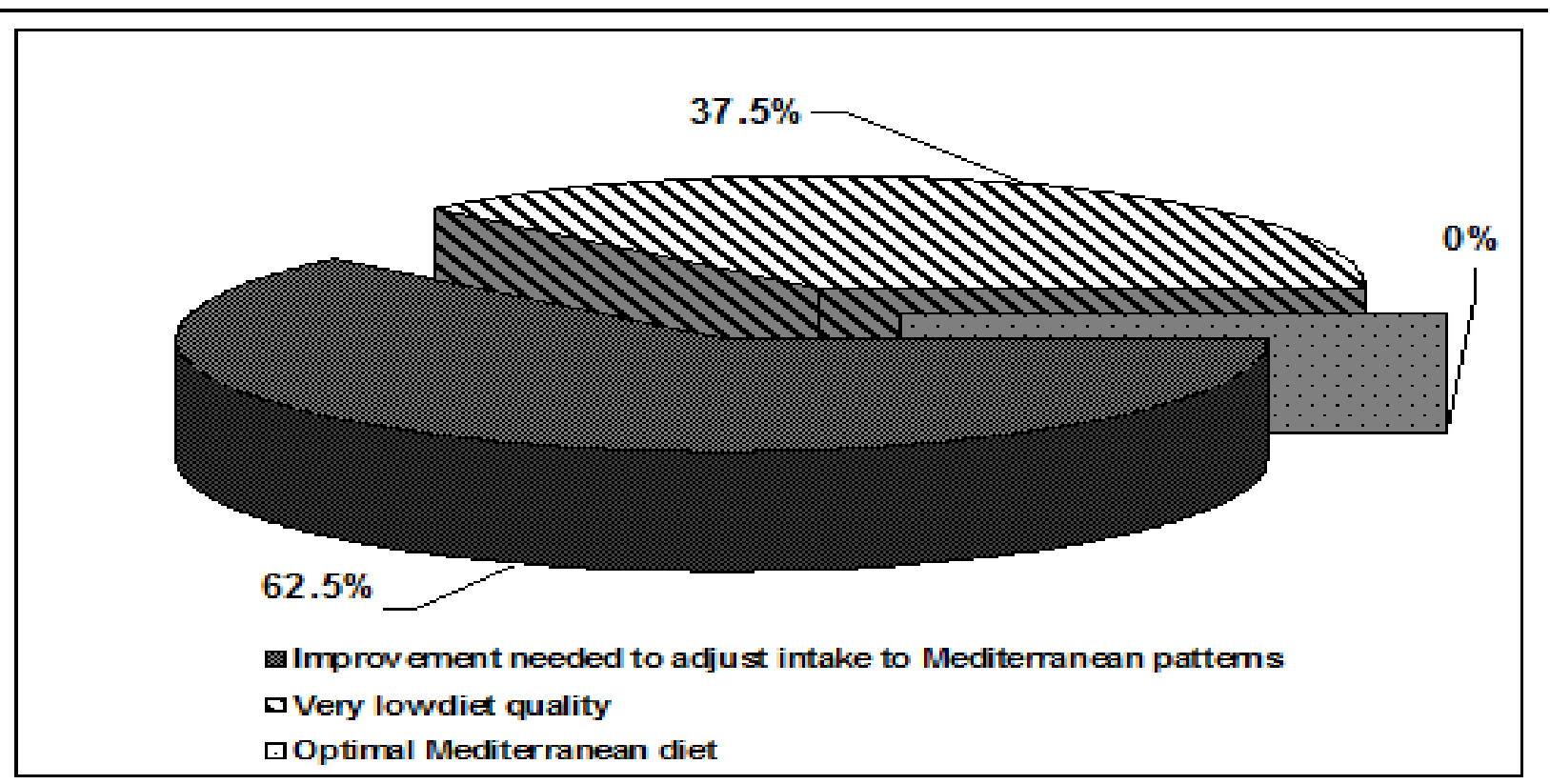

Figure 1: The frequencies of the pupils on the three categories/levels according to the KIDMED test

As it is shown in Figure 1, for the bigger percentage of the pupils with ID (62.5\%) improvement needed to adjust intake to Mediterranean patterns. In addition, the adolescents with intellectual disabilities in a percentage of $37.5 \%$ have a diet of a very low quality. Finally, the proportion of adolescents with ID following optimal Mediterranean diet was zero.

\section{Discussion}

From the results of the present study it was found that the adolescents with ID of the present study have a diet of a very poor quality, or a diet that needs improvement in order to be adjusted to Mediterranean diet patterns. It is worth mentioning that no adolescent of the present sample follows optimal Mediterranean diet. In agreement to the results of the present study, Ptomey (2013) found that adolescents with intellectual and developmental disabilities have a diet that needs improvement, according to Healthy Eating Index (score 57.3). More specifically, the diet intake of adolescents with intellectual and developmental disabilities is high in sodium, very low in dark green and orange vegetables and whole grains, low in total fruit, medium in whole fruit, total vegetables, non-hydrogenated oils and saturated fat, good in total grains, milk, meat and beans, and calories from solid fats, alcoholic beverages and added sugars, but not very good or optimal in any food group. Besides, the lower adherence to a Mediterranean diet has been found to be associated with attention-deficit/hyperactivity disorder diagnosis (RíosHernández et al., 2017).

In similar findings resulted a research that examined data from overweight adults with intellectual and developmental disabilities who participated in a weight loss program (Ptomey et al., 2013). Adults with intellectual and developmental disabilities consumed diets low in fruits, whole grains, and healthy oils, meeting $25.2 \%, 23.0 \%$, and 
$1.1 \%$ of the federal dietary guidelines, respectively. These individuals also consumed a diet high in sodium with $87.3 \%$ consuming more than the recommended intake of sodium (0.7 grams per 1000 kcals). Additionally, they had a lower Healthy Eating Index score than the general population (45.6 vs. 58.2, respectively), with lower scores for fruits, vegetables, meat and beans, oils, and sodium, indicating poor quality of diet for subjects with intellectual and developmental disabilities vs a diet that needs improvement for the general population (Ptomey et al., 2013).

In agreement the adolescents with ID in the present study in a percentage of $37.5 \%$ have a diet of very low quality. In addition, the diet for the bigger percentage of the adolescents $(62.5 \%)$ needs improvement in order to be adjusted to Mediterranean patterns. However, only $25 \%$ of the pupils skip the breakfast, indicating that breakfast was consumed by $75 \%$ of the adolescents with ID. In similar results a research of Ptomey et al., (2013), in adults with intellectual and developmental disabilities, indicating that breakfast was consumed $75 \%$ of the time.

As for eating habits denoting a negative connotation with respect to the Mediterranean diet, the bigger percentage of the adolescents with ID in the present study consume sweets and candy several times a day $(70.8 \%)$, consume commercially baked goods or pastries for breakfast $(62.5 \%)$, and eat more than 1 time/week at a fast-food (hamburger) restaurant (62.5\%). In agreement, Draheim et al. (2007) who assessed the dietary fat intake consumption of adults with ID found that $70.7 \%$ of adults with ID living with family consumed a high-fat diet ( $>30 \%$ of calories from dietary fat). On the contrary, only $29.3 \%$ consumed the recommended $30 \%$ or less of calories from fat. It is worth mentioning that unhealthy dietary patterns (usually high in saturated fat, refined sugars, and processed food and low in fruit and vegetables) are often associated with attention deficit/hyperactivity disorder (Ríos-Hernández et al., 2017).

According to Adolfsson et al. (2008), the main energy sources for adults with ID were milk products, bread, meat products, buns and cakes. The researchers pointed out that the daily intake of fruit and vegetables $(320 \pm 221 \mathrm{~g})$ as well as dietary fiber $(21 \pm 9.6 \mathrm{~g})$ was generally low. The daily intake of fruit and vegetables for the adolescents with ID in the present study is low, too, as $75 \%$ of them take a fruit or a fruit juice every day or/and have a second fruit every day, while $62.5 \%$ have fresh or cooked vegetables $>1$ time/day. On the contrary, $12.5 \%$ of the present sample do not consume any fruit or fruit juice daily and $20.8 \%$ do not consume any fresh or cooked vegetables daily.

Concerning fruit and vegetable consumption of adults with ID living with family, Draheim et al. (2007), also, found that the $58.2 \%$ consumed less than three fruits and vegetable daily. On average subjects with ID consume $1.0 \pm 1.2$ servings of vegetables, $2.8 \pm 2.6$ servings of fruit, and 1.6 \pm 1.9 servings of dairy (Braunschweig et al., 2004). It is worth mentioning that no community-dwelling adults or living with family members consumed the recommended five servings of fruits and vegetables per day (Braunschweig et al., 2004; Draheim et al., 2007). Thus, daily intake of fruits and vegetables for subjects with ID is far away from the recommended servings. Moreover, daily intake of fruits and vegetables for adults with ID is, also, far away from the observed in adults without ID. More specifically, adults without ID in USA consume the 
recommended daily fruits and vegetables in percentages from $17 \%$ to $40 \%$ (Guenther et al., 2006; Thompson et al., 1999).

In addition, in Europe of the 28 Member States, on average, more than half of the general population aged 15 and over (51.4\%) reported that they consume from one to four portions of fruit and vegetables per day, one in seven persons (14.1\%) consume 5 portions of fruit and vegetables or more per day, while more than $1 / 3(34.4 \%)$ do not consume fruits and vegetables on a daily basis. However, the daily consumption of fruit and vegetables differed widely across the EU-28. Thus, in Greece, 1/3 (30\%) of the population aged 15 and over do not consume fruits and vegetables on a daily basis, $62 \%$ consume from one to four portions of fruit and vegetables per day, while only $8 \%$ consume 5 portions of fruit and vegetables or more per day, that is the recommended fruits and vegetables per day (Eurostat, 2018). Consequently, it is of concern that the percentage of adults with ID consuming adequate fruits and vegetables is considerably less than that of adults in the general population (Draheim et al., 2007).

Moreover, Adolfsson et al., (2008) examined diets of 32 adults with ID in Sweden. They found that the diets were low in fiber, vitamin A, thiamin, riboflavin, folic acid, iron, and selenium, and the majority of the daily calories came from milk products, bread, meat, and sweets. In addition, most food was consumed as snacks.

Taking into account the results of other researches as well as the findings of the present study, it could be said that the subjects with ID, adults, youth, as well as adolescents, have a poor diet quality. However, poor diet is not just an observation. Poor nutritional intake is believed to contribute to the increased risk of obesity. In fact, the prevalence of obesity in both adults and adolescents with intellectual and developmental disabilities is approximately 2-3 times greater than in the general population (Ptomey, 2013).

Poor diet, together with physical inactivity, are risk factors for numerous conditions that affect overall health and quality of life, and many of these conditions can lead to chronic diseases. Intermediate outcomes such as obesity, metabolic syndrome, inadequate bone health, undernutrition, iron deficiency, eating disorders, and dental caries can begin in childhood, leading to earlier onset of disease and subsequent premature death (CDC, 2011).

On the other side, engaging children and adolescents in healthy eating and regular physical activity can lower their risk for obesity and related chronic diseases. The major goals in healthy eating are to provide appropriate calorie intake, provide optimum nutrition for the maintenance of health and normal growth, and to help the child develop and sustain healthful eating habits (Daniels et al., 2005).

Concerning Mediterranean diet, it is one of the healthiest eating patterns. Mediterranean diet is associated with lower rates of chronic morbidity and higher life expectancy. The Mediterranean diet showed favorable effects on lipoprotein levels, endothelium vasodilatation, insulin resistance, metabolic syndrome, antioxidant capacity, myocardial and cardiovascular mortality, and cancer incidence in obese patients and in those with previous myocardial infarction (Serra-Majem et al., 2004) 
In addition, it has been proven that far fewer children and youth with special health care needs (CYSHCN) have a healthy weight, while they are much more overweight or obese in comparison with their peers without special health care needs. More specifically, according to the Results of the National Survey on Children's Health (2011/2012) aiming on comparison of CYSHCN with non-CYSHCN ages 0-17 years in Montana, in terms of child's weight status, it was observed that the percentages for CYSHCN were: underweight $6 \%$, healthy weight $55.1 \%$ and overweight or obese $38.8 \%$, while for non- CYSHCN were: underweight $6 \%$, healthy weight $69 \%$ and overweight or obese $25 \%$.

Consequently, as the rates of obesity are higher in individuals with ID than in the general population, special attention should be given to the results of the present study as they suggest high consumption of refined grains and sugar, and low consumption of whole fruits, vegetables, whole grains and fish, and a diet that needs improvement in order to be adjusted to Mediterranean diet patterns, all of which may contribute to the development of diabetes, cardiovascular disease, cancer or other health complications. It is worth mentioning that weight of adults with intellectual and developmental disabilities has been find to be significantly associated with fruit consumption, total vegetables consumption, dark green and orange consumption, and whole grains consumption, such that as the weight of participants is increased, consumption of these foods is decreased (Ptomey et al., 2013). Thus, engaging adolescents with ID in healthy eating in general, and in Mediterranean diet in particular is a cornerstone.

\section{Conclusions}

Consequently, priority should be given to the application and implementation of health educational programs on diet in general, and more specifically on the benefits of Mediterranean diet to adolescents with ID, in order to adopt the Mediterranean diet patterns aiming on the beneficial effects, both directly and long-term in their adult life. It is worth mentioning that the implementation of Mediterranean diet programs in a school environment targeting adolescents at a time in their lives when they develop health attitude behaviors, may be effective throughout their lives.

\section{Conflicts of interest}

The authors declare that there are no conflicts of interest.

\section{References}

Adolfsson, P., Sydner, Y. M., Fjellström, C., Lewin, B., Andersson, A. (2008). Observed dietary intake in adults with intellectual disability living in the community. Food Nutr Res, 52: 10. doi: 10.3402/fnr.v52i0.1857

Alberti-Fidanza A., Fidanza F. (2004). Mediterranean adequacy index of Italian diets. Public Health Nutrition, 7: 937-941. 
Braunschweig, C. L., Gomez, S., Sheean, P., Tomey, K. M., Rimmer, J., Heller, T. (2004). Nutritional status and risk factors for chronic disease in urban-dwelling adults with Down syndrome. American Journal of Mental Retardation, 109(2): 186-193.

CDC (Centers for Disease Control and Prevention). (2011). School Health Guidelines to Promote Healthy Eating and Physical Activity. Morbidity and Mortality Weekly Report, Recommendations and Reports, 60(5): 1-75. U.S. Government Printing Office: 2011.

Daniels, S., Arnett, D., Eckel, R., Gidding, S. S., Hayman, L. L., Kumanyika, S., Robinson, T. N., Scott, B. J., Jeor, S. St., Williams, C. L. (2005). Overweight in children and adolescents: pathophysiology, consequences, prevention, and treatment. Circulation, 111: 1999-2012.

Donini, L., Serra-Majem, L., Bulló, M., Gil, Á., Salas-Salvadó, J. (2015). The Mediterranean diet: Culture, health and science. The British journal of nutrition, 113: S1-S3. $10.1017 /$ S0007114515001087.

Doody, C., Doody, O. (2013). Health promotion for people with intellectual disability and obesity. British Journal of Nursing, 21(8). DOI: 10.12968/bjon.2012.21.8.460.

Draheim, C. C., Stanish, H. I., Williams, D. P., McCubbin, J. A. (2007). Dietary intake of adults with mental retardation who reside in community settings. American Journal of Mental Retardation, 112(5): 392-400.

Ells, L. J., Lang, R., Shield, J. P., Wilkinson, J. R., Lidstone, J. S., Coulton, S., Summerbell, C. D. (2006). Obesity and disability - a short review. Obes Rev, 7(4): 341-345. doi:10.1111/j.1467-789X.2006.00233.x.

Eurostat. (2018). Fruit and vegetable consumption statistics. Statistics explained. https://ec.europa.eu/eurostat/statistics-explained/pdfscache/68501.pdf

Fisher, K., Ketti, P. (2005). Aging with mental retardation: increasing population of older adults with MR require health interventions and prevention strategies. Geriatrics 60(4): 26-29.

Guenther, P. M., Dodd, K. W., Reedy, J., Krebs-Smith, S. M. (2006). Most Americans eat much less than recommended amounts of fruits and vegetables. Journal of the American Dietetic Association, 106: 1371-1379.

Haveman, M., Heller, T., Lee, L., Maaskant, M., Shooshtari, S., Strydom, A. (2010). Major health risks in aging persons with intellectual disabilities: An overview of recent studies. J Pol Pract Intellect Disabil, 7(1): 59-69.

Janicki, M. P., Davidson, P. W., Henderson, C. M., McCallion, P., Taets, J. D., Force, L. T., Sulkes, S. B., Frangenberg, E., Ladrigan, P. M. (2002). Health characteristics and health services utilization in older adults with intellectual disability living in community residences. J Intellect Disabil Res, 46(Pt 4): 287-298.

Jobling, A. (2001). Beyond sex and cooking: health education for individuals with intellectual disability. Ment Retard, 39(4): 310-321.

Marshall, D., McConkey, R., Moore, G. (2003). Obesity in people with intellectual disabilities: the impact of nurse-led health screenings and health promotion activities. J Adv Nurs, 41: 147-153. 
Mokdad, A. H., Marks, J. S., Stroup, D. F., Gerberding, J. L. (2004). Actual causes of death in the United States, 2000. JAMA, 291(10): 1238-1245.

National Survey of Children's Health- NSCH. (2011/12). Data query from the Child and Adolescent Health Measurement Initiative, Data Resource Center for Child and Adolescent Health website. Retrieved September 2016 from www.childhealthdata.org.

Ptomey, L. (2013). Strategies to promote weight loss in adolescents with intellectual and developmental disabilities. Doctoral Dissertation, University of Kansas.

Ptomey, L., Goetz, J., Lee, J., Donnelly, J., Sullivan, D. (2013). Diet Quality of Overweight and Obese Adults with Intellectual and Developmental Disabilities as Measured by the Healthy Eating Index-2005. Journal of Developmental \& Physical Disabilities, 25(6): 625-636. Doi:10.1007/s10882-013-9339-z

Ríos-Hernández, A., Alda, J. A., Farran-Codina, A., Ferreira-García, E., Izquierdo-Pulido, M. (2017). The Mediterranean diet and ADHD in children and adolescents. Pediatrics, 139(2). DOI: 10.1542/peds.2016-2027.

Serra-Majem, L., Ribas, L., Ngo, J., Ortega, R., Garcia, A., Perez-Rodrigo, C., Aranceta, J. (2004). Food, youth and the Mediterranean diet in Spain. Development of KIDMED, Mediterranean Diet Quality Index in children and adolescents. Public Health Nutrition, 7(7): 931-935. Doi: 10.1079/PHN2004556.

Thompson, B., Demark-Wahnefried, W., Taylor, G., McClelland, J. W., Stables, G., Havas, S., Feng, Z., Topor, M., Heimendinger, J., Reynolds, K. D., Cohen, N. (1999). Baseline fruit and vegetable intake among adults in seven 5 a day study centers located in diverse geographic areas. Journal of the American Dietetic Association, 99: 1241-1248.

Trichopoulou, A., Kouris-Blazos, A., Wahlqvist, M. L., Gnardellis, C., Lagiou, P., Polychronopoulos, E., Vassilakou, T., Lipworth, L., Trichopoulos, D. (1995). Diet and overall survival in elderly people. British Medical Journal, 311(7018): 14571460. 
Chrisi Mavrovounioti, Eleni Fotiadou, Eirini Koidou, Souzana Papadopoulou, Eirini Argiriadou MEDITERRANEAN DIET AND ADOLESCENTS WITH INTELLECTUAL DISABILITIES

Creative Commons licensing terms

Authors will retain the copyright of their published articles agreeing that a Creative Commons Attribution 4.0 International License (CC BY 4.0) terms will be applied to their work. Under the terms of this license, no permission is required from the author(s) or publisher for members of the community to copy, distribute, transmit or adapt the article content, providing a proper, prominent and unambiguous attribution to the authors in a manner that makes clear that the materials are being reused under permission of a Creative Commons License. Views, opinions and conclusions expressed in this research article are views, opinions and conclusions of the author(s). Open Access Publishing Group and European Journal of Special Education Research shall not be responsible or answerable for any loss, damage or liability caused in relation to/arising out of conflict of interests, copyright violations and inappropriate or inaccurate use of any kind content related or integrated on the research work. All the published works are meeting the Open Access Publishing requirements and can be freely accessed, shared, modified, distributed and used in educational, commercial and non-commercial purposes under a Creative Commons Attribution 4.0 International License (CC BY 4.0). 